\title{
Evacuation Dynamics Of Asymmetrically Coupled Pedestrian Pairs
}

\author{
Frank Müller and Andreas Schadschneider
}

\begin{abstract}
We propose and analyze extended floor field cellular automaton models for evacuation dynamics of inhomogeneous pedestrian pairs which are coupled by asymmetric group interactions. Such pairs consist of a leader, who mainly determines the couple's motion and a follower, who has a defined tendency to follow the leader. Examples for such pairs are mother and child or two siblings of different age. We examine the system properties and compare them to the case of a homogeneous crowd. We find a strong impact on evacuation times for the regime of strong pair coupling due to the occurrence of a clogging phenomenon. In addition we obtain a non-trivial dependence of evacuation times on the followers' coupling to the static floor field, which carries the information of the shortest way to the exit location. In particular we find that systems with fully passive followers, who are solely coupled to their leaders, show lower evacuation times than homogeneous systems where all pedestrians have an equal tendency to move towards the exit. We compare the results of computer simulations with recently performed experiments.
\end{abstract}

\section{Introduction}

Human crowds and pedestrian traffic are usually composed of both social groups and individuals. Recent empirical studies brought to attention that in this context social groups are rather the normality than the exception [1]. Moussaid et al. [2] observed in a field study that up to $70 \%$ of pedestrians walk in social groups and $\mathrm{Xi}$ et al. [3] found that most pedestrians walk in two-person-groups whereas individual pedestrian traffic is only second frequent. The high relevance of two-person-groups shows the importance of a deeper understanding of the impact such groups impose on evacuation processes.

Frank Müller · Andreas Schadschneider Institut für Theoretische Physik, Universität zu Köln, 50937 Köln, Germany, e-mail: fm@thp. uni-koeln.de, asethp.uni-koeln.de 
We recently introduced models for evacuation processes including social groups which are inspired by methods of non-equilibrium physics [4]. From the perspective of physics the pivotal characteristic of these models is that social groups are cohesive, i.e. group members tend to maintain a spatial coherence. The current work focuses on asymmetrically coupled two-person-groups and aims to provide models for such pairs in evacuation processes. Being the smallest social group two-persongroups still can be very diverse. The strength and symmetry of interaction as well as the group members' level of orientation can differ significantly. Thus it has to be verified which set of models is applicable for simulations. The model types studied here can e.g. describe pairs like mother and child or siblings of different age where one part will dominantly determine the motion and the other part will have a defined tendency to follow. The dominating part will be called leader and the following part will be called follower. The proposed models are used for computer simulations and characteristic effects will be discussed.

The basic underlying model used for the computer simulations is the floor field cellular automaton model (FFCA). It is a stochastic model defined on a 2-dimensional grid with time evolution in discrete steps. A cell can be either empty or occupied by a particle representing a pedestrian. Particles can move by transition to a neighboring cell. The transition will take place with a transition probability arising from different floor fields which encode the tendency to move towards the room's exit and the interaction between pedestrians - here the group cohesion. Further details and general properties of the FFCA can be found in [4].

The evacuation simulations are performed on a standard grid of $63 \times 63$ cells with a moderate pedestrian density of $\rho=0.02$. Observables are averaged over at least 500 runs.

\section{The DGFF and MTFF as mediators of group interaction}

The DGFF and MTFF are the central components which create the group cohesion in the models studied here. We introduced both concepts in [4] and will recap the most important properties in the following.

\subsection{Properties of the DGFF}

In the first proposed model the DGFF mediates the interaction between a leader and the follower. A FFCA with DGFF provides a model for crowds with two-persongroups which have a bond with a likelihood to permanently break up in higher densities.

The DGFF extends the dynamic floor field (DFF) introduced in [5] in several respects. It shares the basic idea that pedestrians increase a field value in the cell they leave when moving to a neighboring cell while decay and diffusion can modify 
it over time. This accounts for the important property that the DGFF is not related to position, but to the movement the leader has performed in preceding time steps. Each couple interacts via its individual DGFF. Only when the leader moves the field is built up and only the follower who is associated with the respective leader reacts on this leader's DGFF. If we do not take the SFF into account followers are most likely to transit to cells with a high associated DGFF value. This causes group members to tend moving on the same trajectory. The full transition probability for the follower including the SFF and the DGFF is

$$
p_{i j}^{F(s)}=N \exp \left(k_{S}^{F} S_{i j}\right) \exp \left(k_{D}^{F} D_{i j}^{F(s)}\right)\left(1-\eta_{i j}\right) \xi_{i j} .
$$

Here $p_{i j}^{F(s)}$ is the transition probability for the follower of pair $s, k_{S}^{F}$ is the coupling constant to the static floor field for followers and $k_{D}^{F}$ is the coupling constant to the DGFF for followers, determining the coupling strength to this field. $N$ is a normalization term. The product $\left(1-\eta_{i j}\right) \xi_{i j}$ guarantees the exclusion principle and avoids transition into wall cells, see e.g. [5].

The transition probability for the leader only considers the SFF, which encodes the shortest way to the exit:

$$
p_{i j}^{L(s)}=N \exp \left(k_{S}^{L} S_{i j}\right)\left(1-\eta_{i j}\right) \xi_{i j}
$$

The DGFF can be understood as a field composed of field quanta (bosons 1 ) with defined internal degrees of freedom. E.g. each field quantum carries the information by which particle it was produced. This allows particles to interact only with bosons of a special type. In particular it enables a follower particle to ignore all bosons, but these of its leader. The leader particles do not react with any boson type. This way an asymmetric group interaction can be established while self-interaction of particles is completely avoided.

It is an important characteristic of the DGFF concept that a moving particle increases the DGFF by $m \gg 1$ instead of only $m=1$ as it is the case in [5]. Small values of $m$ would not lead to sufficiently structured boson traces the followers could continuously follow since only one particle will contribute to the DGFF whereas in case of the DFF all particles contribute to the field. In addition a diffusion parameter $\alpha>0$ is important for continuous group cohesion as it broadens the boson trace. Both factors highly increase the probability that followers do not lose the tracks of their leaders. Figure 1a illustrates the strong dependency of the average distance between leaders and followers $d$ on the diffusion parameter $\alpha$ and the boson multiplicity $m . d$ is a measure for group cohesion. While $m=1$ does not create any noticeable pair bond, $m=400$ causes strong group cohesion.

\footnotetext{
${ }^{1}$ Despite the denomination, "bosons" should not be considered as quantum mechanical particles.
} 


\subsection{Construction of the Moving Target Floor Field}

As explained in Section 2.1 the DGFF is solely increased in a cell when the leader leaves the cell by moving to a neighboring cell. Thus the DGFF is depending on the leader's movement. The question arises how evacuation dynamics changes when the underlying floor field is solely depending on the leader's position. This is the case with the second model we examine here - the moving target floor field (MTFF).

In the FFCA model with MTFF group cohesion is achieved by an asymmetric interaction related to the relative position of the leader with respect to the follower. Every leader of pair $s$ induces a group-specific floor field $M^{(s)}$ in the von Neumann neighborhood of his associated follower:

$$
M_{i j}^{(s)}(T)=\max _{(\tilde{i}, \tilde{j})}\left\{\sqrt{\left(i_{L}(T)-\tilde{i}\right)^{2}+\left(j_{L}(T)-\tilde{j}\right)^{2}}\right\}-\sqrt{\left(i_{L}(T)-i\right)^{2}+\left(j_{L}(T)-j\right)^{2}}
$$

$\left(i_{L}(T), j_{L}(T)\right)$ denotes the position of the leader at time step $T$. The first term on the r.h.s. is a normalization term where $(\tilde{i}, \tilde{j})$ runs over the cells in the von Neumann neighborhood of the follower.

The MTFF contributes to the transition probabilities in an analogous manner as the DGFF in Section 2.1. The total transition probability of the follower $F$ of pair $s$ is

$$
p_{i j}^{F(s)}=N \exp \left(\left(k_{S}^{F} S_{i j}+k_{M} M_{i j}^{(s)}\right)\left(1-\eta_{i j}\right) \xi_{i j} .\right.
$$

\section{Impact of pair cohesion on evacuation dynamics}

In this section we will investigate the question how the fragmentation of a pedestrian crowd into asymmetrically coupled pedestrian pairs impacts evacuation dynamics and how the resulting evacuation process compares to the scenario with a homogeneous crowd without pair bonds. We will analyze both DGFF and MTFF systems and compare the results.

\subsection{Comparison of DGFF, MTFF and homogeneous model}

For the purpose of comparison with a homogeneous crowd the model is configured such that leaders and followers are equipped with the same level of orientation, which is realized by an equal coupling constant with respect to the SFF. When $k_{D}^{F}=0$ the configuration $k_{S}^{L}=k_{S}^{F}$ coincides with a homogeneous crowd with no interaction between the pedestrians.

First we turn to the model with DGFF. For growing $k_{D}^{F}$ the homogeneous crowd is fragmented into asymmetrically coupled pairs which increasingly maintain proximity and tend to move on the same trajectory. How will this impact evacuation 
dynamics? Figure $1 \mathrm{~b}$ shows the dependence of $T$ on $k_{D}^{F}$ for the DGFF model: The coupling in pairs slightly improves the evacuation process. The effect is small, but it is clearly visible that $T$ drops for growing $k_{D}^{F}$. The drop takes place in a comparably small interval since the boson multiplicity is high at $m=400$. It is interesting to note that the effect does not coincide with a lower average number of conflicts per time step. An analysis of this number shows that conflicts are even increased for $k_{D}^{F}>0$, but still $T$ is lowered. The higher number of conflicts is due to the group cohesion. The continuous proximity of group members increases the likelihood that these choose the same cell for a transition which leads to an overall increase of conflicts.

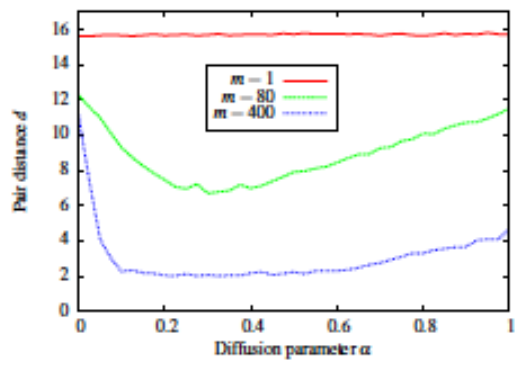

(a)

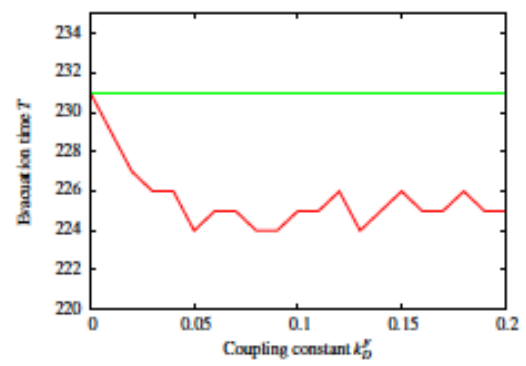

(b)

Fig. 1: Different views on the DGFF model. (a) Dependence of the average pair distance $d$ on the diffusion parameter $\alpha$ for different values of the boson multiplicity $m$. (b) Dependence of $T$ on $k_{D}^{F}$. The dashed line refers to $T=T_{\text {hom }}$, which is the average evacuation time of a homogeneous crowd without pair bonds.

The improvement of evacuation time $T$ in this model becomes comprehensible when recalling the nature of the DGFF. It encodes spatio-temporal information about the path the leader has successfully moved on - not only spatial information about the leader. Without movement no DGFF builds up. Therefore the information contributes to choose successful paths through the crowd.

The coupling mechanism of the MTFF highly differs from that of the DGFF since it is not related to the movement of the leader, but to his position. In fact simulations show that this difference translates to measurable differences in the respective evacuation processes. Figure $2 \mathrm{a}$ is the analogon of Figure $1 \mathrm{~b}$ for the MTFF model. It shows a non-trivial dependence of the evacuation time $T$ on the coupling parameter $k_{M}$. The dashed line in Figure 2a refers to the average evacuation time $T$ of a homogeneous system without pair bonds. In both figures coupling to the SFF is at $k_{S}^{L}=k_{S}^{F}=0.8$. In the domain of $k_{M} \lesssim 0.6$ the coupling in pairs results in a lower $T$ while for $k_{M} \gtrsim 0.6 T$ is increased. $T$ remains nearly constant for $k_{M} \gtrsim 4(T \approx 258)$ until clogging processes increase $T$ again. Clogging is discussed in Section 3.3 . 


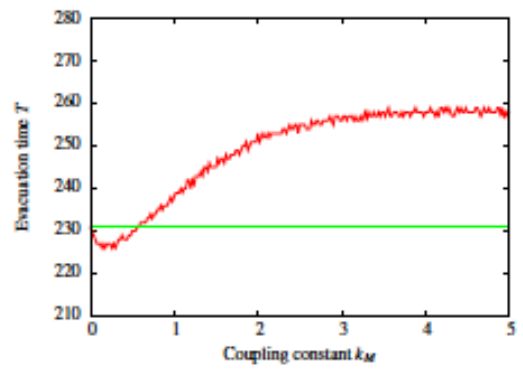

(a)

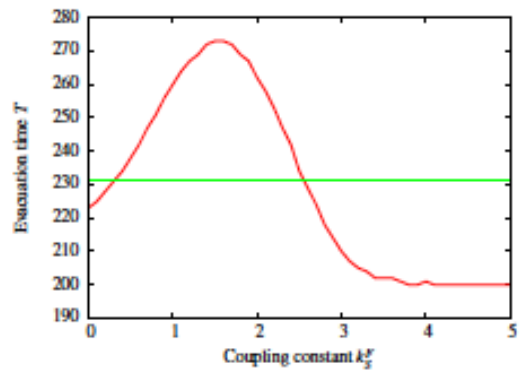

(b)

Fig. 2: Different views on the average evacuation time $T$ for systems with MTFF. The dashed line visualizes $T=T_{\text {hom }}$, which is the average evacuation time of a homogeneous crowd without pair bonds and $k_{S}=0.8$. (a) Dependence of evacuation time $T$ on the coupling strength $k_{M}$ in a configuration with $k_{S}^{L}=k_{S}^{F}=0.8$. (b) Dependence of evacuation time $T$ on $k_{S}^{F}$ which controls the follower's coupling to the SFF. Pair coupling strength is constant at $k_{M}=2$ and $k_{S}^{L}=0.8$.

\subsection{Influence of the follower's coupling to the static floor field}

In this section we shift the point of interest to the question how the coupling of the follower to the static floor field (SFF) $k_{S}^{F}$ influences the evacuation process. Apart from the coupling to the leader via MTFF the follower is also coupled to the SFF, which encodes the shortest way to the exit. $k_{S}^{F}=0$ means that the follower's motion is not oriented at the exit at all whereas $k_{S}^{F} \rightarrow \infty$ leads to a deterministic movement on the shortest path to the exit. $k_{S}^{F}$ can be interpreted as the follower's orientation towards the exit or more generally as the ability and will to reach the exit himself.

Figure $2 \mathrm{~b}$ shows the resulting average evacuation time for a system with $k_{M}=2$. The dashed line depicts the evacuation time $T_{h o m}$ of the homogeneous reference system without pair coupling and equal $k_{S}$. It is a counterintuitive result that fully passive followers, who do not have any tendency to move to the exit themselves lead to a more efficient evacuation with lower evacuation times than a homogeneous crowd with an equally good orientation towards the exit. It appears to be beneficial if followers are solely led by their leaders. In contrary strong pair coupling together with the equally good orientation towards the exit $\left(k_{S}^{L}=k_{S}^{F}=0.8\right)$ slows down the evacuation. This result was also found in Figure $2 \mathrm{a}$

The domain of $k_{S}^{F} \gtrsim 2.5$ where $T$ falls below $T_{\text {hom }}$ again arises from a situation where followers have such good ability to reach the exit themselves that despite their pair bond they overtake their leaders and reach the exit first. Here the overall average evacuation time benefits from the fast evacuation of the followers. 


\subsection{Clogging and gridlocks}

In [4] we had found clogging phenomena for the asymmetric fixed-bond leaderfollower model. In this model the pair bond is fully fixed as for every cell transition the follower is positioned on the cell the leader had occupied previously. The pair distance is always $d=1$. The question remained if such clogging phenomena can be found when the bond is dynamic and $d$ can take on arbitrary values. Indeed this is the case for systems with MTFF.

In Section 3.1 it was addressed that for high $k_{M}$ and the resulting high group cohesion clogging starts to increase the average evacuation time $T$. Figure 3 a shows such increase. Here $T$ fluctuates strongly and the standard deviation of $T$ is accordingly high. The phenomenon occurs due to followers who maintain the nearest possible position to their leaders in front of the exit and impede their leaders from reaching the exit. If a configuration occurs where followers completely shield their leaders from exiting no particle will be evacuated until the situation dissolves. Figure $3 \mathrm{~b}$ displays an example of such situations. For very high values of $k_{M}$ the shielding followers have a nearly vanishing probability to ever leave the cell next to their leader and it comes to a final stop of the evacuation (gridlock). This is a situation which is not found in reality. However, clogging due to pairs who do not let go each other in a high density situation is well conceivable and a possible source of impediment in evacuation processes through a narrow door. The high standard deviation in the domain of clogging indicates that evacuation scenarios become increasingly unpredictable once clogging becomes a likely effect during the evacuation.

The described clogging phenomenon does not occur in systems with DGFF as this field is solely built up by the motion of the respective leader. While the MTFF only encodes spatial information about the leader the DGFF encodes spatio-temporal information about the path the leader has successfully moved on. When it comes to

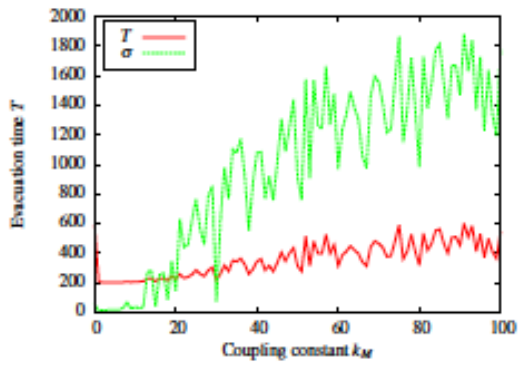

(a)

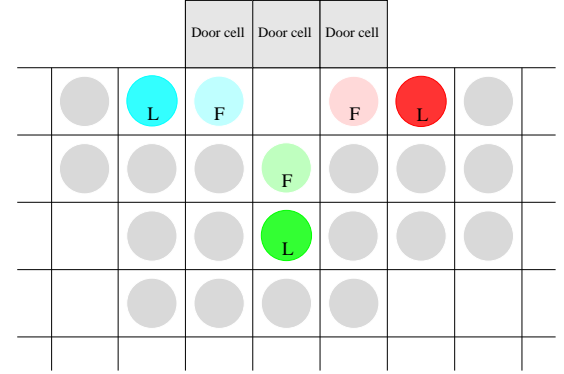

(b)

Fig. 3: Clogging in systems with MTFF. (a) Dependence of evacuation time $T$ on the coupling strength $k_{M}$ with $k_{S}^{L}=0.8$ and $k_{S}^{F}=0.2$. High values of $k_{M}$ lead to increased values of $T$ and high $\sigma(T)$ due to clogging. (b) Example for a typical clogging situation for a system with door width $d=3$. 
highly congested states the DGFF is only rarely increased and the decay mechanism brings the field strength down to low values or zero. Then movement is mainly governed by the SFF and particles start moving towards the exit again. Thus systems with DGFF cannot develop long-term clogging or even gridlocks.

\section{Conclusion}

Quantitatively for moderate coupling both models show only small deviations from the average evacuation time $T$ of a homogeneous reference system without pair coupling. This result is in line with the evacuation experiments we recently performed with students [6]. However, qualitatively the two models differ significantly from each other as the DGFF model always leads to a decreased $T$ while the MTFF model shows two domains with decreased $T$ for low and increased $T$ for high coupling strength $k_{M}$. At present the collected data from experiment does not provide sufficient statistical significance to rank one model over the other. Further experiments will contribute to investigate this question.

For the MTFF system our simulations have shown a non-trivial dependency of $T$ on $k_{S}^{F}$. The fragmentation of a crowd into couples with fully passive followers results in a more efficient evacuation process than a homogeneous crowd.

In the domain of strong coupling to the MTFF clogging leads to an increase of $T$ and a high standard deviation, which makes the average evacuation time $T$ less meaningful and a single evacuation process less predictable. This is an important factor when simulations are to predict evacuation times, e.g. for evacuation assistants to support decisions about optimum evacuation routes during emergencies.

Acknowledgements We dedicate this contribution to the memory of our friend and colleague Matthias Craesmeyer. Financial support by the DFG under grant SCHA 636/9-1 is gratefully acknowledged.

\section{References}

1. V.L. Knoop and W. Daamen, editors. Traffic and Granular Flow '15. Springer, in press.

2. M. Moussaïd, N. Perozo, S. Garnier, D. Helbing, and G. Theraulaz. The walking behaviour of pedestrian social groups and its impact on crowd dynamics. PLOS ONE, 5(4):e10047, 2010.

3. J. Xi, X. Zou, Z. Chen, and J. Huang. Multi-pattern of complex social pedestrian groups. Transportation Research Procedia, 2:60 - 68, 2014.

4. F. Müller, O. Wohak, and A. Schadschneider. Study of influence of groups on evacuation dynamics using a cellular automaton model. Transportation Research Procedia, 2:168 - 176, 2014.

5. A. Kirchner and A. Schadschneider. Simulation of evacuation processes using a bionicsinspired cellular automaton model for pedestrian dynamics. Physica A, 312:260-276, 2002.

6. C. von Krüchten, F. Müller, A. Svachiy, O. Wohak, and A. Schadschneider. Emperical study of the influence of social groups in evacuation scenarios. In Traffic and Granular Flow '15, in press. 